\title{
A Robust Dual Watermarking Technique for Medical Images in E- Healthcare Records
}

\author{
${ }^{* 1}$ Lendale Venkateswarlu, ${ }^{2} \mathrm{~N}$ Vyagreswara Rao, ${ }^{3} \mathrm{~B}$ Eswara Reddy \\ ${ }^{* 1}$ Research Scholar, JNTUA, Anantapuram, CMR College of Engg. \& Tech., Medchal (Dist)., TG, India. \\ ${ }^{2}$ Department of CSE, CVR College of Engineering, Ibrahimpatan, R.R. Dist., TG, India. \\ ${ }^{3}$ Department of CSE, JNTUA College of Engineering, Kalikiri, Chittoor Dist., AP, India \\ Email: *1venkatlendale@gmail.com, ${ }^{2}$ nvr@ieee.org, ${ }^{3}$ eswarcsejntua@gmail.com
}

\section{Received: 20 ${ }^{\text {th }}$ November 2017, accepted: 02 ${ }^{\text {nd }}$ December 2017, Published: 28th February 2018}

\begin{abstract}
This paper proposes a prudent implementation of robust pipeline in the domain of watermark based secure medical image transmission. The pipeline comprises of three stages, involving insertion of first watermark in the first stage and the second watermark in the second stage. Insertion of the watermark invokes a multi transform algorithm implemented in two steps. The first step is the application of Two-Way Arnold Transformation (TWAT) which scrambles the pixel locations in the source image, based on the number of cycles passed as one of the arguments to it, leading to a reduction in spatial correlation. The second step is 2-D Wavelet Transformation (WT) applied to the scrambled pixels that exploit the reduced correlation feature to generate the large values of coefficients. These large coefficient values can efficiently accommodate the wavelets coefficients of the inserted watermark. This step is a novel contribution compared to the existing models. Subsequently, the inverse WT is applied which concludes the first stage of the pipeline. The second stage of the pipeline also implements the same sequence of steps as that of the first stage but with the second watermark. In the final stage of the pipeline, the inverse TWAT is applied twice the number of times to the forward transformations applied in the first two stages. The entire pipeline is termed as Multi Transform based Robust Dual Water Marking (MTRDW). The pipeline is implemented on benchmark images with patient information and hospital logo as watermarks. The claimed features of the model are presented through computing the quality metrics such as SSIM, NCC and PSNR from the experiments conducted.
\end{abstract}

Keywords: DWT, Integrity, Medical Imaging, PSNR, SSIM, TWAT, Watermarking

\section{Introduction}

The Information technology applications in biomedicine are combined with the hospital environment medical data resulting into online dispensary which is also known as telemedicine [1]. As a natural consequence, maintaining the confidentiality, integrity and robustness of data on the Internet against the various malicious attacks has become a complex task [2-4]. In the scenario of Networked Hospitals [5-11], exchanging medical information among the hospitals is a common phenomenon. Medical information (informatics) is generally presented in the form of images [12-13].
Medical Imaging is a process of creating images of the parts of the human body for the purposes of prognostic diagnosis purpose [14]. Medical imaging techniques such as endoscopy, cardiology, radiology, ultrasound scan etc. are used to study the functioning of various parts of the human body.

Telemedicine and image processing reduce the difficulty involved in exchanging medical images among hospitals manually. But, the distribution of medical information over the Internet poses a threat of illegal use of the information. Therefore, the security of medical image is a prime goal of medical image processing. Digital watermarking [15-16] techniques are used for providing the security [17] to any medical image which is commonly referred to as the cover image. Digital watermarking is a process of embedding some extra piece of information (watermark) which can be easily retrieved and compared with the original information to assess the authenticity of information. This technique is widely used for adding authentication information and provides additional security without affecting the contents of the cover image. Many researchers have proposed various digital watermarking techniques that are classified based on various parameters such as watermarking domain, type of document on which watermark is applied, human perception (vision and speech) and the applications. Broadly speaking there are two types of watermarking techniques: Visible and Invisible. Visible watermarking techniques render the watermark signal to be clearly visible in the cover image to mark the ownership of the content. The invisible watermarking inserts the watermark in such a way that it is not visible to the human eye. Thus, the invisible watermark is used to provide image authentication and protect the image from being duplicated. According to the literature, digital watermarking techniques should possess the following properties:

Transparency - The inserted watermark should not reduce the quality of the cover image.

Robustness - The watermark should be designed in such a way that, it is unaffected by all attacks.

Capacity - Capacity defines the maximum amount of data that can be inserted into image without degrading the cover image.

Fidelity - Fidelity refers to the similarity of original and watermarked images. Watermarking should not 
introduce visible distortions as it reduces the correctness of the data.

Security - Secret key must be used for embedding and detection process [18-22].

While inserting the watermark, care must be taken so that watermark is embedded in the proper Region of Non-Interest (RONI) [23-24]. Otherwise, crucial information pertaining to the patient may be lost [2530]. Any information pertaining to a patient such as patient identity as watermark helps in preserving the integrity of data related to a specific patient. Many researchers have proposed watermarking techniques but very few have attempted in dual watermarking as it involves fidelity of the image. The first double watermarking technique was proposed by Wen-Nung Lie in 2003[50]. Subsequently, Bo Chen [51] proposed a dual robust fragile watermarking technique. Li Jing and W. X. Wang proposed dual zero watermarking [5253]. Very few researchers have proposed a double watermarking technique in medical images. Keeping all these aspects in view, a robust dual copyright watermarking technique is developed and presented in this paper.

Section 2 describes the basic theory of the two transformations used in the technique; Section 3 contains the details of the method employed. Results and analysis are described in detail in section 4 .

\section{Fundamental Theory of Transforms}

The two transformations used in the proposed watermarking techniques are

1. Two-way Arnold transformation

2. Discrete Wavelet transformation.

Two -Way Arnold transformation

In the literature of image scrambling techniques, there are many innovative algorithms to scramble an image [31-32]. Two-Way Arnold Transformation (TWAT) algorithm [33-35] is a recent development in scrambling techniques, which scrambles the image in two directions at the same time. If we take an image $\mathrm{NxN}$ with $(\mathrm{x}, \mathrm{y})$ as original pixel coordinates then new coordinates (X', Y') are obtained by the following transformation.

$$
\begin{aligned}
& {\left[\begin{array}{l}
X^{\prime} \\
Y^{\prime}
\end{array}\right]=\left[\begin{array}{ll}
2 & 3 \\
3 & 5
\end{array}\right]\left[\begin{array}{l}
x \\
y
\end{array}\right] \operatorname{MOD}(N) \text { Where } \quad \mathrm{x}, \quad \mathrm{y} \quad €} \\
& \{1 \ldots \ldots \ldots . . \mathrm{N}\} .
\end{aligned}
$$

This transformation contributes to the distribution of pixels of medical images uniformly that affects the brightness of the image. But the inverse of this transformation makes it possible to restore the image to its original state.

Application of TWAT once to the original image is considered as one cycle. If it is applied twice, then it is called two cycles. Every application of TWAT to the Arnold transformed image brings the more uniform distribution of pixels in the image. In Fig. 1 Two Way Arnold Transformation of chest Image is shown.
Decryption of n-cycle TWAT Image requires the application of Inverse Two Way Arnold Transformation (ITWAT) n-times.

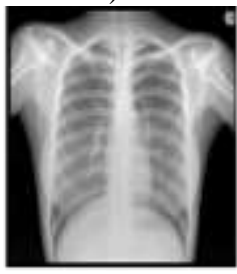

(a) 0-Cycles

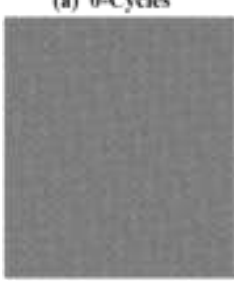

(d) 5-Cycles

(g) 20-Cycles

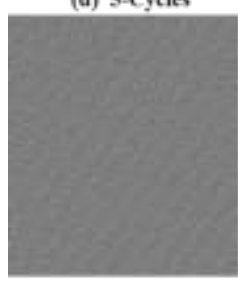

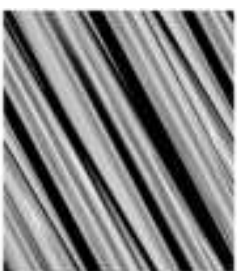

(b) 1-Cycles

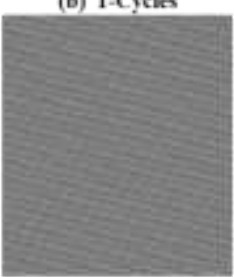

(c) 10-Cycles

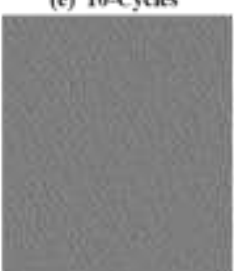

(h) 25-Cycles

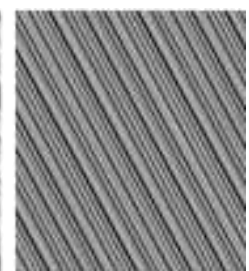

(c) 2-Cycles

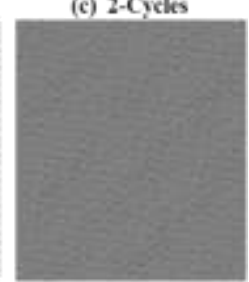

(f) 15-Cycles

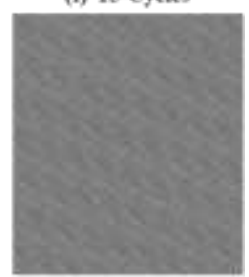

(i) 30-Cycles
Fig. 1 (a) - (i): Resultant Images after applying Twoway Arnold Transformation with Varying Cycles.

\section{Discrete Wavelet Transformation}

The Discrete wavelet transformation (DWT) of the original image produces four sub-band images [36]. A cover image, say I, can be represented by a 2Dimensional array $\mathrm{I}[\mathrm{m}, \mathrm{n}]$ with $\mathrm{m}$ rows and $\mathrm{n}$ columns where $\mathrm{m}, \mathrm{n}$ are positive integers. DWT is first applied on $\mathrm{m}$ rows of the cover image to get low-frequency (L) and high-frequency $(\mathrm{H})$ bands. Information about the edges is present in high-frequency, whereas the lowfrequency bands are again split into low-frequency and high-frequency sub-bands. Since the human eye is less sensitive to visua

lize the change in edges, the high-frequency components are used in watermarking. DWT can be applied row and column wise to get DWT coefficients such as LL1, HL1, LH1, and HH1. They are also known as sub-bands as shown in Fig. 2. LL1 sub-band can be further subdivided into four more sub-bands as shown in the Fig. 2... 


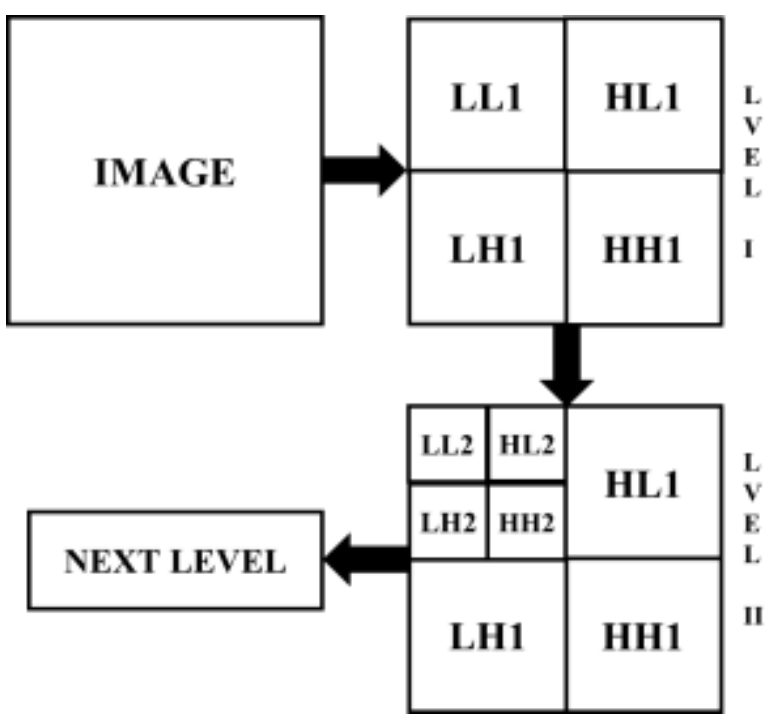

Fig. 2: Two-level Wavelet Transform

This process of subdivision can be continued to the required number of levels. The frequency sub-band $\mathrm{LH}$ contains the vertical information of the cover image, HL contains the horizontal information of the cover image and the $\mathrm{HH}$ sub-band contains the diagonal information of the cover image [37-41]. Very often watermark is inserted in LH and HL bands because these bands produce a less distorted watermarked image

\section{Proposed Two Way Arnold and Wavelet Based Robust Dual Watermarking Method}

Original Robust dual copyright watermarking [42-48] technique for medical image involves two aspects.

1. Dual watermark insertion

2. Dual watermark extraction

The dual watermarks used for medical images are hospital logo (HLOGO) and patient identification (PID). Dual watermarking is a process of embedding two watermarks for transmission of images in a secure way. Two invisible watermarks (HLOGO, PID) are embedded into a medical image. Generally, these dual watermarks have an advantage of robustly carrying the dual marks relating to the owner. The results are tested with quality metrics such as Structural Similarity Index (SSIM)[49], Normalized Cross Correlations (NCC) and Peak Signal to Noise Ratio (PSNR)

\subsection{Dual watermark insertion}

Dual watermark insertion algorithm is the stepwise procedure to insert watermarks HLOGO and PID. It invokes the single watermark insertion algorithm twice to insert a watermark. Because the cover image is scrambled two times watermarks do not overlap with each other.

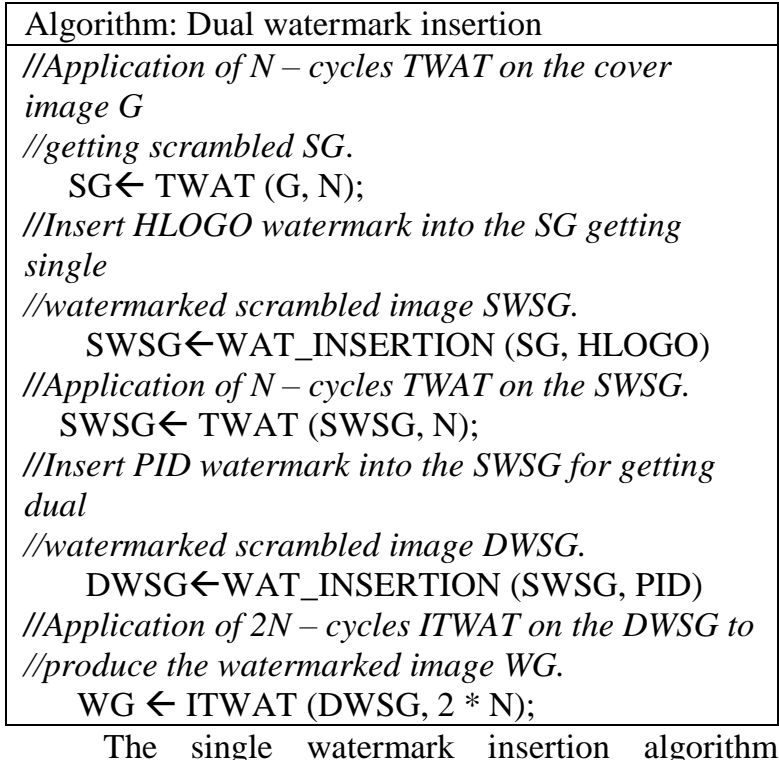
describes the steps involved in watermark insertion. In this algorithm to insert the watermarks are performed by applying the formula:

$\mathrm{F}(\mathrm{m}, \mathrm{n})=\mathrm{f}(\mathrm{m}, \mathrm{n})+\alpha * \mathrm{~W}(\mathrm{i}, \mathrm{j})$ eq. 1

where $0<\alpha<=1$ is a scaling, factor used for embedding watermark. $\mathrm{F}(\mathrm{m}, \mathrm{n})$ represents scrambled watermarked wavelet coefficients and $f(m, n)$ are the scrambled cover image wavelet coefficients. W $(i, j)$ is the matrix of Wavelets coefficient of the image in the frequency domain.

\begin{tabular}{|l|}
\hline Algorithm: Single watermark insertion \\
\hline Procedure WAT_INSERTION (SG, W) \\
//SG- is scrambled cover image \\
$/ / W$-is watermark image \\
BEGIN \\
//Apply DWT on the SG getting four different bands of \\
$/ /$ Wavelet coefficients. \\
[SGLL SGLH SGHL SGHH] $\leftarrow$ DWT(SG); \\
//Apply DWT on the W. \\
[WLL WLH WHL WHH] $\leftarrow$ DWT(W) \\
//scale down the Wavelet coefficients of W by using \\
$/ /$ scaling factor $\alpha$ \\
[WLL WLH WHL WHH] $\leftarrow \alpha *[W L L$ WLH WHL \\
WHH] \\
//Add the W content in SGLH band. \\
ADD (SGLH, WLL) \\
//Apply inverse DWT on SG** for getting watermarked \\
$/ /$ image WSG. \\
WSG $\leftarrow$ IDWT (SGLL, SGLH, SGHL, SGHH) \\
END.
\end{tabular}

A step by step process for dual watermarking insertion is shown in Fig. 3. 


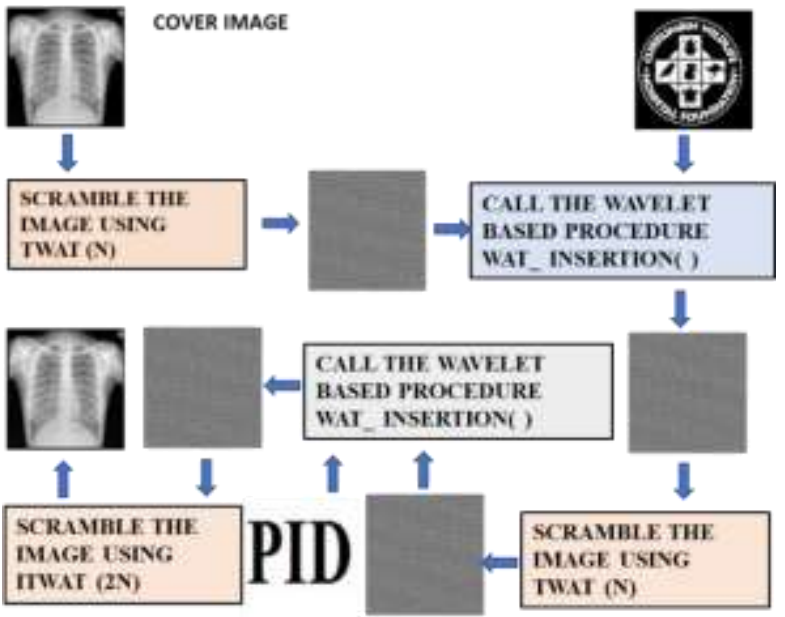

Fig. 3: Dual Watermark insertion

Dual Watermark extraction

In the watermark extraction technique, the watermarked image is first scrambled by applying the TWAT. Watermarked image and the original cover image are split into same $\mathrm{N}$ levels that were done for embedding the watermark. For retrieving the watermark, the original image should be known for extraction. This kind of a watermarking technique calls Non-Blind watermarking technique. The extraction procedure is given by:

$$
\mathrm{W}(\mathrm{i}, \mathrm{k})=(\mathrm{FR}(\mathrm{m}, \mathrm{n})-\mathrm{f}(\mathrm{m}, \mathrm{n})) / \alpha ; \ldots \ldots . . \text { eq. } 2
$$

Where FR $(m, n)$ are DWT coefficients of received watermarked image, $f(m, n)$ is the DWT coefficients of the original cover image after scrambling using TWAT. $\alpha$ is a scaling factor applied to the insertion of a dual watermark. As noise may be added to the image by attacks or transmission over the public or private network channel to communicate, the extracted sequence may contain both positive and the negative values due to noise present in the communication channel.

A step by step procedure for dual watermark extraction is shown in Fig.4



Fig. 4: Watermarking Extraction
The same is given as an algorithm consisting of the following steps to retrieve the dual watermark.

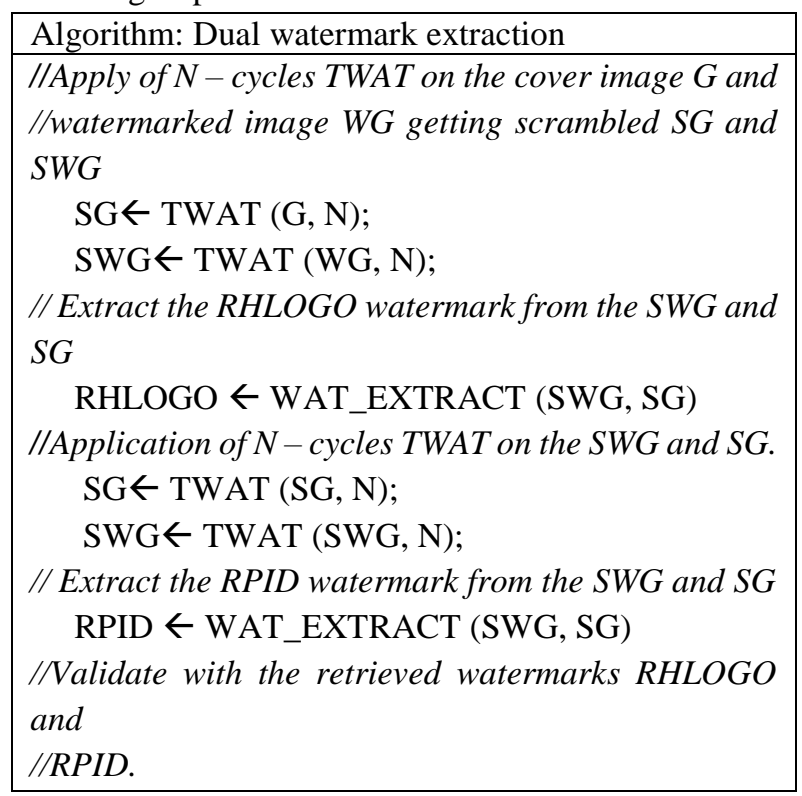

Dual watermark extraction algorithm explains step by step procedure to retrieve the two watermarks. It calls the single watermark extraction algorithm two times to retrieve the watermarks.

\begin{tabular}{l}
\hline Algorithm: Single watermark extraction \\
\hline Procedure WAT_EXTRACTION (WSG, SG) \\
//SG, WSG- is scrambled cover image and \\
watermarked \\
//image \\
BEGIN \\
//Apply DWT on the SG and WSG getting four \\
different \\
//bands of Wavelet coefficients. \\
[WSGLL WSGLH WSGHL WSGHH] \\
DWT(WSG); \\
[SGLL SGLH SGHL SGHH] $\leftarrow$ DWT(SG); \\
//Retrieve the WLL content in SGLH band. \\
RETRIVE (SGLH, WLL...) \\
$/ /$ scale up the Wavelet coefficients of WLL... by using \\
$/ /$ scaling factor $\alpha$ \\
[WLL WLH WHL WHH] $\leftarrow$ [WLL WLH WHL \\
WHH] / $\alpha$ \\
$/ /$ Apply inverse DWT on WLL... for getting \\
watermark \\
//image W. \\
W $\leftarrow$ IDWT (WLL...) \\
END.
\end{tabular}

\section{Experimental Results}

The proposed algorithm is simulated using MATLAB code with forty-five medical cover images (size 512X512) and nine watermark images (size $128 \mathrm{X}$ 128 ) as input. First, the cover image is a medical image and is scrambled by applying TWAT instead of directly 
applying DWT. To insert watermarks, TWAT is applied with varying iterations to scramble the medical image.

The dual watermarking technique is simulated with various attacks like noise (Gaussian Noise), compression (Jpeg Compression) and cropping (Geometric Cropping along left, right, Down and up) using MATLAB. For demonstrating the work two medical images are considered. Fig.5 (a) chest X-Ray and Fig.5 (b) cross-sectional image of a human skull is the original cover images.

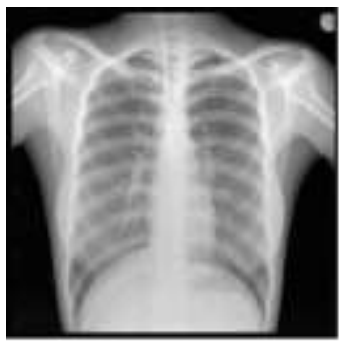

(a)



(b)
Fig. 5: Cover images (a) chest X-Ray and (b) CrossSectional Image of a human skull.

Fig. 6 (a) and 6(b) are the resultant images after applying the TWAT to the two cover images eleven times.



(a)

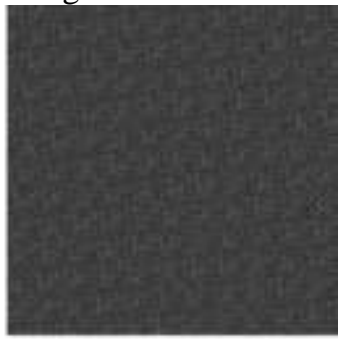

(b)
Fig. 6: Transformed cover images: (a) chest X-Ray and (b) Cross-Sectional Image of human skull The Fig.7 (a) \& 7(b) are the dual watermarked images of chest X-ray and CT-scan respectively. It is observed that perceptually cover images in Fig. 5 and watermarked images in Fig.7 are identical. Therefore, it is concluded that inserting a watermark on images does not perceptually deteriorate original image, which is a necessary condition for medical images. Experimental results show high PSNR values for watermarked images and extracted watermarks.

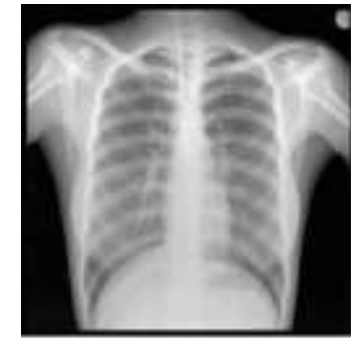

(a)

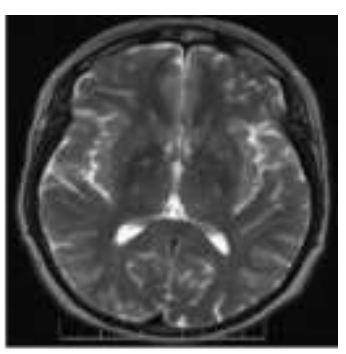

(b)
Fig. 7 (a) and (b) Dual watermarked images
Images in Fig. 8 show the watermark images and their respective extracted images before attacks which demonstrates that the watermarks are perceptually identical.

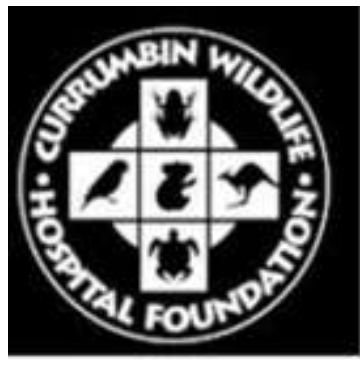

(a)

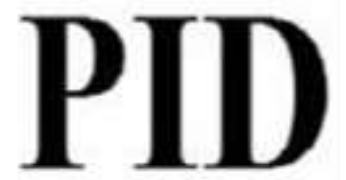

(c)



(b)

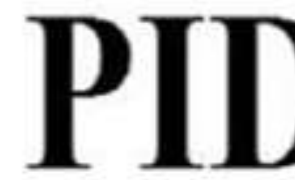

(d)

Fig. 8 (a) and (c) original watermarks, (b) and (d) extracted watermarks

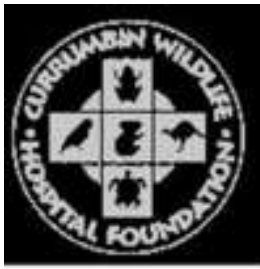

(a)

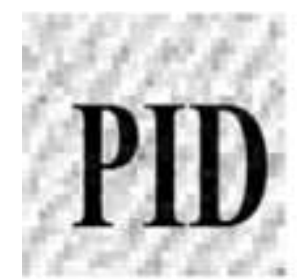

(d)



(b)

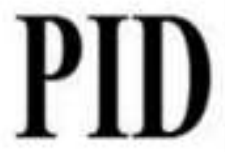

(e)

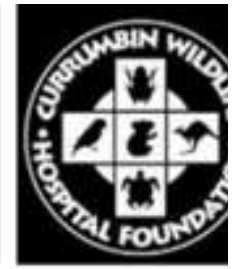

(c)

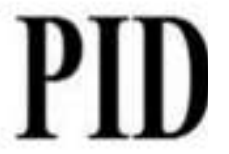

(f)
Fig. 9 Extracted Watermarks after attacks. Figs 9(a) and 9(d) show the images extracted after the Gaussian attack with a variance of 0.0001 . Figs 9(b) and 9(e) shows the images extracted after $10 \%$ Compression attack, Fig 9(c) and 9(f) are the images extracted after $2 \%$ (x and y axis) cropping attack

Tables I, II...VIII shows the experimental results for the images in terms of metrics for the quality of images with various scales. The metrics considered are Peak Signal Noise Ratio (PSNR) structural similarity (SSIM) index and Normalized Cross Correlation (NCC).

Table I Shows quality metrics of X-Ray and CT-Scan images before and after various attacks (Gaussian, 
Compression and Cropping attacks). From the values, it is clear that as watermark content increases the quality of cover image decreases. But this decrease is not hampering perceptual visibility of the cover image.

TABLE I watermarked images with varying scaling factors

\begin{tabular}{|l|l|l|l|l|l|}
\hline \multirow{2}{*}{$\begin{array}{l}\text { Cover } \\
\text { image }\end{array}$} & \multicolumn{5}{|l|}{ Watermarked image } \\
\cline { 2 - 6 } & \multirow{2}{*}{ Metrics } & $\begin{array}{l}\alpha= \\
0.01\end{array}$ & $\begin{array}{l}\alpha= \\
0.05\end{array}$ & $\begin{array}{l}\alpha= \\
0.1\end{array}$ & $\begin{array}{l}\alpha= \\
0.15\end{array}$ \\
\hline \multirow{3}{*}{ X-Ray } & PSNR & 54 & 42 & 36 & 32 \\
\cline { 2 - 6 } & SSIM & 1.0 & 0.96 & 0.856 & 0.733 \\
\cline { 2 - 6 } & NCC & 1.0021 & 1.0025 & 1.0028 & 1.0030 \\
\hline \multirow{3}{*}{$\begin{array}{l}\text { CT- } \\
\text { Scan }\end{array}$} & PSNR & 54 & 41 & 35 & 32 \\
\cline { 2 - 6 } & SSIM & 0.997 & 0.954 & 0.862 & 0.765 \\
\cline { 2 - 6 } & NCC & 1.0022 & 1.0025 & 1.0027 & 1.0027 \\
\hline
\end{tabular}

Table II shows the values of PSNR, SSIM and NCC after extracting the watermark from the image. It contains quality metrics for extracted watermarks with different scaling factors. The values in tables show that the inserted and extracted watermarks are almost identical which is evident from high PSNR values for the watermark.

TABLE II Extracted watermark with varying scaling factors

\begin{tabular}{|l|l|l|l|l|l|}
\hline \multirow{2}{*}{$\begin{array}{l}\text { Watermark } \\
\text { image }\end{array}$} & \multicolumn{4}{|l|}{ Extracted Watermark } \\
\cline { 2 - 7 } & metrics & $\begin{array}{l}\alpha= \\
0.01\end{array}$ & $\begin{array}{l}\alpha= \\
0.05\end{array}$ & $\begin{array}{l}\alpha= \\
0.1\end{array}$ & $\begin{array}{l}\alpha= \\
0.15\end{array}$ \\
\hline \multirow{3}{*}{ HLOGO } & PSNR & $\mathrm{H}$ & $\mathrm{H}$ & $\mathrm{H}$ & $\mathrm{H}$ \\
\cline { 2 - 7 } & SSIM & 1 & 1 & 1 & 1 \\
\cline { 2 - 6 } & NCC & 1.001 & 1.001 & 1.001 & 1.001 \\
\hline \multirow{3}{*}{ PID } & PSNR & $\mathrm{H}$ & $\mathrm{H}$ & $\mathrm{H}$ & $\mathrm{H}$ \\
\cline { 2 - 7 } & SSIM & 1 & 1 & 1 & 1 \\
\cline { 2 - 7 } & NCC & 1.001 & 1.001 & 1.001 & 1.001 \\
\hline
\end{tabular}

Table III shows the values of quality metrics when the watermarked image is subjected to Gaussian noise attack with variance $0.0001,0.0005,0.001$ and 0.0015 when the scaling factor is fixed as 0.1 . It is evident from tables that quality parameters (PSNR, SSIM and NCC) drastically decreased when watermarked image is subjected to Gaussian attack.

TABLE III Gaussian noise attack on watermarked image with varying variance

\begin{tabular}{|c|c|c|c|c|c|}
\hline \multirow{2}{*}{$\begin{array}{l}\text { Cover } \\
\text { image }\end{array}$} & \multicolumn{5}{|c|}{$\begin{array}{l}\text { Watermarked image fixed with Scaling factor } \\
0.1\end{array}$} \\
\hline & metrics & $\begin{array}{l}\sigma^{2}= \\
0.0001\end{array}$ & $\begin{array}{l}\sigma^{2}= \\
0.0005\end{array}$ & $\begin{array}{l}\sigma^{2}= \\
0.001\end{array}$ & $\begin{array}{l}\sigma^{2}= \\
0.0015\end{array}$ \\
\hline \multirow{3}{*}{$\begin{array}{l}X- \\
\text { RAY }\end{array}$} & PSNR & 28 & 26 & 20.4 & 20.4 \\
\hline & SSIM & 0.287 & 0.286 & 0.285 & 0.286 \\
\hline & $\mathrm{NCC}$ & 1.0024 & 1.0031 & 1.0034 & 1.0037 \\
\hline \multirow{3}{*}{$\begin{array}{l}\text { CT- } \\
\text { SCAN }\end{array}$} & PSNR & 20.4 & 20.4 & 20.4 & 20.4 \\
\hline & SSIM & 0.218 & 0.216 & 0.218 & 0.216 \\
\hline & NCC & 1.0025 & 1.0032 & 1.0035 & 1.0039 \\
\hline
\end{tabular}

Table IV shows quality metrics of extracted watermark images HLOGO and PID with variance 0.0001, 0.0005, 0.001 and 0.0015 when the scaling factor is fixed as 0.1 . The results are encouraging in terms of robustness and quality of the watermark images against Gaussian noise attack.
TABLE IV Extracted watermark when Gaussian noise attack applied on watermarked image

\begin{tabular}{|l|l|l|l|l|l|}
\hline \multirow{2}{*}{$\begin{array}{l}\text { Watermark } \\
\text { image }\end{array}$} & \multicolumn{5}{|l|}{ Extracted watermark fixed with scaling 0.1} \\
\cline { 2 - 6 } & metrics & $\begin{array}{l}\sigma^{2}= \\
.0001\end{array}$ & $\begin{array}{l}\sigma^{2}= \\
0.0005\end{array}$ & $\begin{array}{l}\sigma^{2}= \\
0.001\end{array}$ & $\begin{array}{l}\sigma^{2}= \\
0.0015\end{array}$ \\
\hline \multirow{3}{*}{ HLOGO } & PSNR & 20.5 & 19.4 & 20.2 & 19.5 \\
\cline { 2 - 6 } & SSIM & 0.898 & 0.873 & 0.874 & 0.89 \\
\cline { 2 - 6 } & NCC & 0.892 & 0.881 & 0.879 & 0.875 \\
\hline \multirow{3}{*}{ PID } & PSNR & 19.5 & 18.2 & 18.6 & 18.6 \\
\cline { 2 - 6 } & SSIM & 0.732 & 0.675 & 0.634 & 0.625 \\
\cline { 2 - 6 } & NCC & 0.870 & 0.866 & 0.872 & 0.861 \\
\hline
\end{tabular}

Table V shows quality metrics when compression attack is applied on the watermarked image. The percentage of compression is taken as 5,10,15 and 20. It is evident from the values that quality parameters are decreased when watermarked image is subjected to Jpeg compression attack.

TABLE V Watermarked image with Jpeg Compression attack is applied

\begin{tabular}{|l|l|l|l|l|l|}
\hline \multirow{2}{*}{$\begin{array}{l}\text { Cover } \\
\text { image }\end{array}$} & \multicolumn{5}{|c|}{ Watermared image with compression is applied } \\
\cline { 2 - 6 } & metrics & $\begin{array}{l}\text { Comp }= \\
5 \%\end{array}$ & $\begin{array}{l}\text { Comp }= \\
10 \%\end{array}$ & $\begin{array}{l}\text { Comp }= \\
15 \%\end{array}$ & $\begin{array}{l}\text { Comp= } \\
20 \%\end{array}$ \\
\hline \multirow{2}{*}{$\begin{array}{l}\text { X- } \\
\text { RAY }\end{array}$} & PSNR & 33.9 & 33.9 & 34.5 & 34.9 \\
\cline { 2 - 6 } & SSIM & 0.819 & 0.822 & 0.842 & 0.853 \\
\cline { 2 - 6 } & NCC & 0.977 & 0.977 & 0.978 & 0.979 \\
\hline \multirow{2}{*}{$\begin{array}{l}\text { CT- } \\
\text { SCAN }\end{array}$} & PSNR & 33.7 & 34.1 & 35.4 & 36.4 \\
\cline { 2 - 6 } & SSIM & 0.846 & 0.858 & 0.890 & 0.910 \\
\cline { 2 - 6 } & NCC & 0.980 & 0.980 & 0.982 & 0.983 \\
\hline
\end{tabular}

Table VI shows quality metrics of extracted watermark images HLOGO and PID when Jpeg compression attack with the percentage of compression taken as $5,10,15$ and 20 is applied. The results are encouraging in terms of robustness and quality of the watermark images against the attack.

TABLE VI Extracted watermark when Jpeg compression attack applied on watermarked image

\begin{tabular}{|c|c|c|c|c|c|}
\hline \multirow{2}{*}{$\begin{array}{l}\text { Watermark } \\
\text { image }\end{array}$} & \multicolumn{5}{|c|}{$\begin{array}{l}\text { Extracted Watermark with compression is } \\
\text { applied }\end{array}$} \\
\hline & metrics & $\begin{array}{l}\text { Comp } \\
5 \%\end{array}$ & $\begin{array}{l}\text { Comp } \\
10 \%\end{array}$ & $\begin{array}{l}\text { Comp } \\
15 \%\end{array}$ & $\begin{array}{l}\text { Comp } \\
20 \%\end{array}$ \\
\hline \multirow{3}{*}{ HLOGO } & PSNR & $\mathrm{H}$ & $\mathrm{H}$ & 25.0 & 20.7 \\
\hline & SSIM & 1.0 & 1.0 & 0.970 & 0.894 \\
\hline & $\mathrm{NCC}$ & 0.8661 & 0.792 & 0.759 & 0.716 \\
\hline \multirow{3}{*}{ PID } & PSNR & $\mathrm{H}$ & $\mathrm{H}$ & 22.8 & 15.8 \\
\hline & SSIM & 1.0 & 1.0 & 0.631 & 0.548 \\
\hline & $\mathrm{NCC}$ & 0.8671 & 0.794 & 0.759 & 0.712 \\
\hline
\end{tabular}

TABLE VII cropping attack on watermarked

image
\begin{tabular}{|l|l|l|l|l|l|}
\hline $\begin{array}{l}\text { Cover } \\
\text { image }\end{array}$ & \multicolumn{4}{|l|}{ Watermarked image fixed with scaling factor0.1 } \\
\cline { 2 - 7 } & metrics & $\begin{array}{l}\text { Left } \\
10 \%\end{array}$ & $\begin{array}{l}\text { Right } \\
10 \%\end{array}$ & $\begin{array}{l}\text { Top } \\
10 \%\end{array}$ & $\begin{array}{l}\text { Bottom } \\
10 \%\end{array}$ \\
\hline \multirow{2}{*}{$\begin{array}{l}\text { X- } \\
\text { RAY }\end{array}$} & PSNR & 40 & 41 & 40 & 41 \\
\cline { 2 - 6 } & SSIM & 0.89 & 0.89 & 0.89 & 0.89 \\
\cline { 2 - 6 } & NCC & 1.0014 & 1.0014 & 1.0014 & 1.0014 \\
\hline \multirow{2}{*}{$\begin{array}{l}\text { CT- } \\
\text { SCAN }\end{array}$} & PSNR & 40 & 41 & 41 & 41 \\
\cline { 2 - 6 } & SSIM & 0.89 & 0.89 & 0.89 & 0.89 \\
\cline { 2 - 6 } & NCC & 1.0014 & 1.0014 & 1.0014 & 1.0014 \\
\hline
\end{tabular}


Table VII shows the values of quality metrics when the watermarked image is subjected to cropping attack with left, right, top and bottom when the scaling factor is fixed as 0.1. It is evident from tables that quality parameters (PSNR, SSIM and NCC) decreased when watermarked image is subjected to the attack.

Table VIII shows quality metrics of extracted watermark images HLOGO and PID when cropping 10 percentage on left, right, top and bottom when the scaling factor is fixed as 0.1 is applied. The results are encouraging in terms of robustness and quality of the watermark images against the attack.

TABLE VIII Extracted watermark when cropping attack applied on watermarked image

\begin{tabular}{|c|c|c|c|c|c|}
\hline \multirow{2}{*}{$\begin{array}{l}\text { Watermark } \\
\text { image }\end{array}$} & \multicolumn{5}{|c|}{$\begin{array}{l}\text { Extracted watermark fixed with scaling } \\
\text { factor } 0.1\end{array}$} \\
\hline & metrics & $\begin{array}{l}\text { Left } \\
10 \%\end{array}$ & $\begin{array}{l}\text { Right } \\
10 \%\end{array}$ & $\begin{array}{l}\text { Top } \\
10 \%\end{array}$ & $\begin{array}{l}\text { Bottom } \\
10 \%\end{array}$ \\
\hline \multirow{3}{*}{ HLOGO } & PSNR & 20 & 20 & 20 & 20 \\
\hline & SSIM & 0.86 & 0.86 & 0.86 & 0.86 \\
\hline & NCC & 0.901 & 0.901 & 0.901 & 0.901 \\
\hline \multirow{3}{*}{ PID } & PSNR & 20 & 20 & 20 & 20 \\
\hline & SSIM & 0.86 & 0.86 & 0.86 & 0.86 \\
\hline & NCC & 0.901 & 0.901 & 0.901 & 0.901 \\
\hline
\end{tabular}

\section{Conclusion}

The present work describes the concept of dual watermarking. The cover medical image was dual watermarked maintaining high robustness. The two different types of invisible watermarks used were the robust watermarks HLOGO and PID. The process started by applying TWAT to medical images followed by DWT and their inverse. The major part of the work involves the insertion and extraction of invisible watermarks and the outputs were tested by applying different attacks to the output images. The experimental results show the good quality watermarked images. And when attacks are applied, retrieved watermarks were not remarkably degraded. The above findings are evident from the values of the image metrics PSNR, SSIM and NCC.

\section{References}

1. A. Zvikhachevskaya, G. Markarian and L. Mihailova, "Quality of Service Consideration for the Wireless Telemedicine and E-Health Services," 2009 IEEE Wireless Communications and Networking Conference, Budapest, 2009, pp. 1-6.

2. S. Vellaisamy and V. Ramesh, "Inversion attack resilient zero-watermarking scheme for medical image authentication," in IET Image Processing, vol. 8, no. 12, pp. 718-727, 122014.

3. M. T. Naseem, I. M. Qureshi, Atta-ur-Rahman and M. Z. Muzaffar, "Robust watermarking for medical images resistant to geometric attacks," 15th International Multitopic Conference (INMIC), Islamabad, 2012, pp. 224-228.

4. J. M. Zain and A. R. M. Fauzi, "Medical Image Watermarking with Tamper Detection and Recovery," International Conference of the IEEE
Engineering in Medicine and Biology Society, New York, NY, 2006, pp. 3270-3273.

5. S. Kaur, O. Farooq, R. Singhal, B. S. Ahuja, "Digital watermarking of ECG data for secure wireless communication," IEEE Transactions on Telecommunication and Computing, pp. 140-144, 2010.

6. Ahjoku Amadi-Obi, Peadar Gilligan, Niall Owens, Cathal O'Donnell "Telemedicine in pre-hospital care: a review of telemedicine applications in the pre-hospital environment" International Journal of Emergency Medicine, Amadi-Obi. Springer 2014.

7. Ronald S. Weinstein, Ana Maria Lopez, Bellal A. Joseph, Kristine A., Michael Holcomb, Gail P. Barker, Elizabeth A. Krupinski "Telemedicine, Telehealth, and Mobile Health Applications That Work: Opportunities and Barriers" The American journal Medicine, Volume 127, Issue 3, Pages 183187,2014

8. Paul T. Kim, Richard A. Falcone "The use of telemedicine in the care of the pediatric trauma patient" Seminars in Pediatric Surgery Elsevier Volume 26, Issue 1, Pages 47-53, 2017.

9. M. Raza,M. Hoa Le, N. Aslam, C. Hieu Le, N. Tam Le, T. Ly Le "Telehealth Technology: Potentials, Challenges and Research Directions for Developing Countries" IFMBE Proceedings. Springer, p. 233-236, 2016.

10. S. Thelen, M. Czaplik, P. Meisen, D. Schilberg and S. Jeschke, "Using off-the-Shelf Medical Devices for Biomedical Signal Monitoring in a Telemedicine System for Emergency Medical Services," in IEEE Journal of Biomedical and Health Informatics, vol. 19, no. 1, pp. 117-123, Jan. 2015.

11. M. Görs, M. Albert, K. Schwedhelm, C. Herrmann, K. Schilling, "Design of an Advanced Telemedicine System for Remote Supervision," in IEEE Systems Journal, vol. 10, no. 3, pp. 10891097, Sept. 2016.

12. G. Coatrieux and L. Lecornu, "A review of Image Watermarking Applications in healthcare," In proc. 28th Annual International Conference of the IEEE Engineering in Medicine and Biology Society, EMBS '06. 2 pp. 4691-4694, 2006.

13. H. M. Chao, C. M. Hsu, and S. G. Miaou, "A datahidding technique with authentication, integration, and confidentiality for electronic patient records,' IEEE Trans. Inf. Technol. Biomed., vol. 6, no. 1, pp. 46-53, Mar. 2002.

14. R. Eswaraiah and E. Sreenivasa Reddy, "Robust medical image watermarking technique for accurate detection of tampers inside region of interest and recovering original region of interest," in IET Image Processing, vol. 9, no. 8, pp. 615-625, 82015.

15. Cox, M. Miller, J. Bloom, and C. Honsinger, "Digital watermarking," J. Electron. Imaging, vol. 11, no. 3 , p. 414,2002

16. TianruiZong, Yong Xiang, IynkaranNatgunanathan, Song Guo, Wanlei Zhou, GlebBeliakov "Robust histogram shape-based method for image watermarking" IEEE Transactions on Circuits and Systems for Video Technology, vol 25(5), pp717 - 729, 2015.

17. I.F Kallel, M. Kallel and M.S Bouhlel "A secure fragile watermarking algorithm for medical image authentication in the DCT domain" 2nd International Conference on Information \& Communication Technologies, Vol 1, pp. 2019 2024, 2006.

18. H. Abdel-Nabi and A. Al-Haj, "Efficient joint encryption and data hiding algorithm for medical 
images security," 2017 8th International Conference on Information and Communication Systems (ICICS), Irbid, Jordan, 2017, pp. 147-152.

19. Kelkar and H. Nemade, "Reversible watermarking in medical images using Histogram shifting method with improved security and embedding capacity," IEEE International Conference on Recent Trends in Electronics, Information \& Communication Technology (RTEICT), Bangalore, 2016, pp. 971975.

20. W. Ma and K. Sartipi, "Security middleware infrastructure for medical imaging system integration and monitoring," 18th International Conference on Advanced Communication Technology (ICACT), Pyeongchang, 2016, pp. 1-1.

21. Naveen, T. Venkata Sainath Gupta, V. R. Satpute and A. S. Gandhi, "A simple and efficient approach for medical image security using chaos on EZW," Eighth International Conference on Advances in Pattern Recognition (ICAPR), Kolkata, 2015, pp. 16.

22. N. V. Rao, V. Meena Kumari, "Watermarking in Medical Imaging for Security and Authentication" Information Security Journal: A Global Perspective, $T \&$ F, Vol. 20, issue 20,2011.

23. H. K. Lee, H. J. Kim, K. R. Kwon, et al, "Digital watermarking of medical image using ROI information" Enterprise Networking and Computing in Healthcare Industry, proceedings of 7th International Workshop on 23-25, pp. 404-407, June 2005.

24. H. K. Lee, H. J. Kim, K. R. Kwon, et al, "ROI medical image Watermarking using DWT and Bitplane,", Asia-Pacific Conference on Communications, Perth, Western Australia, pp. 512-515, October 2005.

25. M. Moniruzzaman and M. F. Hossain, "Watermarking approach of embedding patient facial information into RONI of Brain $\mathrm{CT}$ scan image," 2015 18th International Conference on Computer and Information Technology (ICCIT), Dhaka, 2015, pp. 248-253.

26. H. Nyeem, W. Boles and C. Boyd, "Utilizing Least Significant Bit-Planes of RONI Pixels for Medical Image Watermarking," 2013 International Conference on Digital Image Computing: Techniques and Applications (DICTA), Hobart, TAS, 2013, pp. 1-8.

27. G. Coatrieux, J. Montagner, H. Huang and C. Roux, "Mixed Reversible and RONI Watermarking for Medical Image Reliability Protection," 29th Annual International Conference of the IEEE Engineering in Medicine and Biology Society, Lyon, 2007, pp. 5653-5656.

28. Singh and M. K. Dutta, "A blind \& fragile watermarking scheme for tamper detection of medical images preserving ROI," International Conference on Medical Imaging, m-Health and Emerging Communication Systems (MedCom), Greater Noida, 2014, pp. 230-234.

29. M. Jamali, S. Samavi, N. Karimi, S. M. R. Soroushmehr, K. Ward and K. Najarian, "Robust watermarking in non-ROI of medical images based on DCT-DWT," 38th Annual International Conference of the IEEE Engineering in Medicine and Biology Society (EMBC), Orlando, FL, 2016, pp. 1200-1203.

30. R. Keshavarzian, "A new ROI and block based watermarking scheme using DWT," 20th Iranian Conference on Electrical Engineering (ICEE2012), Tehran, 2012, pp. 1323-1328.

31. S. Che, Z. Che and B. Ma, "An Improved Image Scrambling Algorithm," Second International
Conference on Genetic and Evolutionary Computing, Hubei, 2008, pp. 495-499

32. B. Radu, D. A. Cristina, P. Iustin and F. Cristina, "A new fast chaos-based image scrambling algorithm," 10th International Conference on Communications (COMM), Bucharest, 2014, pp. 14.

33. Ni Rongrong, RuanQiuqi. "Image Information Hiding Algorithm with the Symetry of Arnold Transform" [J]. Journal of North Jiaotong University,26(2):25-28, 2002.

34. Venkateswarlu Lendale, B. Eswara Reddy, N Vyaghreswara Rao. "Arnold-wavelet based robust watermarking technique for medical images." ICT in Business Industry \& Government (ICTBIG), International Conference on. IEEE, 2016.

35. Y. Su, P. Han, Z. J. Jia, S. H. Liang, "Digital Watermarking Based on Two-Way Arnold Transform and QR Code", Advanced Materials Research, Vols. 591-593, pp. 2564-2567, 2012

36. [Giakoumaki, S. Pavlopoulos, and D. Koutouris, "A medical image watermarking scheme based on wavelet transform in engineering, medicine and biology society", proceedings of the 25th Annual International Conference of the IEEE, vol. 1, pp. $856-859,2003$.

37. P. Fakhari, E. Vahedi, and C. Lucas, "Protecting patient Privacy from unauthorized release of medical images using a bio-inspired wavelet based watermarking approach," Digital Signal Processing, vol. 21, no. 3, pp. 433 - 446, 2011.

38. F. Taher, A. Kunhu and H. AlAhmad, "A new hybrid watermarking algorithm for MRI medical images using DWT and hash functions," 38th Annual International Conference of the IEEE Engineering in Medicine and Biology Society $(E M B C)$, Orlando, FL, 2016, pp. 1212-1215.

39. N. Mettripun, "A robust medical image watermarking based on DWT for Patient Identification," 13th International Conference on Electrical Engineering/Electronics, Computer, Telecommunications and Information Technology (ECTI-CON), Chiang Mai, 2016, pp. 1-6.

40. Kunhu, F. Taher, H. Al-Ahmad, "A new multi watermarking algorithm for medical images using DWT and hash functions," 11th International Conference on Innovations in Information Technology (IIT), Dubai, 2015, pp. 230-234

41. T. Pardhu, B.R. Perli, "Digital image watermarking in frequency domain," 2016 International Conference on Communication and Signal Processing (ICCSP), Melmaruvathur, 2016, pp. 0208-0211.

42. Giakoumaki, S. Pavlopoulos, D. Koutsouris "Secure and efficient health data management through multiple watermarking on medical images" Medical and Biological Engineering and Computing, springer, 2006.

43. A. Giakoumaki, S. Pavlopoulos and D. Koutsouris, "A Multiple Watermarking Scheme Applied to Medical Image Management," The 26th Annual International Conference of the IEEE Engineering in Medicine and Biology Society, 2004, pp. 32413244.

44. Bo Chen and Hong Shen "A new robust-fragile dual image watermarking algorithm" Multimedia and Ubiquitous Engineering, Third International Conference, pp 153-157, 2009.

45. Xingyang Zhao and Jiyin Sun, "A novel third party's double watermarking scheme reject to malice attack," IEEE International Conference on Network Infrastructure and Digital Content, Beijing, 2009, pp. 1018-1022. 
46. B. Chen and H. Shen, "A New Robust-Fragile Double Image Watermarking Algorithm," Third International Conference on Multimedia and Ubiquitous Engineering, Qingdao, 2009, pp. 153157.

47. H. Lu and W. Xia, "A Double Image Watermarking Based on Wavelet Domain and Krawtchouk Moments," International Symposium on Computer Network and Multimedia Technology, Wuhan, 2009, pp. 1-4.

48. L. Venkateswarlu, N. V. Rao and B. E. Reddy, "A Robust Double Watermarking Technique for Medical Images with Semi-fragility," 2017 International Conference on Recent Advances in Electronics and Communication Technology (ICRAECT), Bangalore, India, 2017, pp. 126-131.

49. Z. Wang, A. C. Bovik, H. R. Sheikh, and E. P. Simoncelli, "Image quality assessment: From error visibility to structural similarity" IEEE Trans. Image Process., vol. 13, no. 4, pp. 600-612, Apr. 2004.

50. Wen-Nung Lie, Tze-Liang Hsu and Guo-Shiang Lin, "Verification of image content integrity by using dual watermarking on wavelets domain," Proceedings $2003 \quad$ International Conference on Image Processing (Cat. No.03CH37429), 2003, pp. II-487-90 vol.3.

51. B. Chen and H. Shen, "A New Robust-Fragile Double Image Watermarking Algorithm," 2009 Third International Conference on Multimedia and Ubiquitous Engineering, Qingdao, 2009, pp. 153157.

52. Li Jing and F. Liu, "Double Zero-Watermarks Scheme Utilizing Scale Invariant Feature Transform and Log-Polar Mapping," 2007 IEEE International Conference on Multimedia and Expo, Beijing, 2007, pp. 2118-2121.

53. W. X. Wang, H. L. Xu and S. Li, "Double Zerowatermarking Algorithm on Hyperchaotic Iteration," 2009 Third International Symposium on Intelligent Information Technology Application, Nanchang, 2009, pp. 567-570 